\title{
Quality of Life, Lifestyle Diseases, Mental Health and Cognitive Functioning: A Neuroanthropological Approach
}

\author{
Urvashi Gupta, Shweta Jain, Meenal Dhall* \\ Department of Anthropology, University of Delhi, Delhi-07, India
}

\begin{abstract}
Neuroanthropology considers the complex web of association and action among lifestyle diseases, mental health, cognitive function and quality of life. A flawed lifestyle results in increased levels of risk for high blood pressure, abnormal amounts of cholesterol in the bloodstream, high blood glucose, respiratory diseases, excessive body fat accumulation, and heartrelated diseases. There is psychological well-being that influences how one thinks, feels, acts, handles stress, relates to others and makes choices as well as behavior. A sound lifestyle is instrumental in the promotion of healthy cognitive functioning and effective ageing while deteriorating cognitive function is a hallmark of ageing that predicts mortality. Quality of life is a complex multidimensional concept evaluated by people based on the horizons of possibilities that they see for themselves. Decline in cognitive function from lifestyle habits, environment (both social and physical), chronic diseases tend to raise severe challenges towards maintaining a good quality of life, worsening with poor mental and physical health. Even though people are encouraged to practice healthy habits to prevent most of the chronic medical disorders, they generally ignore or are reluctant to either start or maintain the appropriate and healthy behavior. Domains of all these subsets seem to possess different paradigms and their influence meet upon the same base-human, thereby overlapping in terms of repercussions arising from undesirable undertakings.
\end{abstract}

KEYWORDS: Quality of life; Lifestyle diseases; Mental health; Cognition; Neuroanthropological

\section{INTRODUCTION}

Neuroanthropology has exemplified the holism that has been a feature of anthropology since Franz Boas' work. The area looks to biology and culture, and it welcomes both empiricism and critical analysis. It necessitates interdisciplinary approaches by definition and borrows a focus on the mechanisms and processes underlying cognitive functions from biological anthropology. Virtually all cognitive functions are complex assemblages involving parts of the human brain in relation to external elements, such as learned culture, other people, perceptions of the environment, and

\section{Vol No: 05, Issue: 01}

Received Date: June 26, 2020

Published Date: September 09, 2021

\section{*Corresponding Author}

\section{Dr. Meenal Dhall}

Physiological Anthropology and

Kinanthropometry and ergonomics Laboratory, Department of Anthropology, University of Delhi, Delhi-07, India, Tel: +919650159434

E-mail: say2meenal@gmail.com

Citation: Meenal D, et al. Quality of Life, Lifestyle Diseases, Mental Health and Cognitive Functioning: A Neuroanthropological Approach.

Mathews J Neurol. 5(1):19.

Copyright: Urvashi G, et al. (C) 2021. This is an open-access article distributed under the terms of the Creative Commons Attribution License, which permits unrestricted use, distribution, and reproduction in any medium, provided the original author and source are credited. 
material culture. Neuroanthropologists realize that elements of "cognitive systems" can be found in the environment [1]. Decline in cognitive function is a result of external and internal factors inclusive of lifestyle habits, social and physical environment as well as other chronic diseases, which in turn raises severe challenges to maintain good quality of life worsening with poor mental and physical health. Despite significant advances in the understanding of the neurobiology of cognitive decline and dementia, there are still no obvious determinants or full causal models for explaining the risks associated with these conditions. Significant evidence suggests that certain lifestyle choices (particularly smoking, sedentary lifestyle and poor dietary choices) are associated with a faster rate of cognitive decline and a higher risk of dementia, while physical, mental, and social leisure activities are protective [2].

Quality of life, term as defined by the World Health Organization as, an individual's perception of their position in life in the context of the culture and value systems in which they dwell in firm association with their goals, expectations, standards and concerns sustenance [3]. It is a complex multidimensional concept evaluating both positive and negative aspects of life, with a high degree of subjectivity. Even though the term itself contains meaning for nearly everyone but can be defined differently by groups and individuals making it challenging to measure [4]. So, quality of life is the degree to which an individual considers themselves healthy, comfortable and able to participate in/enjoy life events, referring to both the experience an individual has of his/her own life and to the living conditions in which individuals find themselves. When one person may define quality of life according to the wealth that they bear or by satisfaction with life, another person may address it in terms of capabilities like having the ability to lead a good life that is both emotionally and physically sound. It is interesting to note here that a disabled person may report a high quality of life, whereas a healthy person who recently lost a job may respond to being undergoing a low quality of life [5]. It is attributed to the multidimensional nature of the term that encompasses emotional, physical, material and social wellbeing considered together. Along with health determining the overall quality of life, there are other domains as well such as aspects of job, housing, school, neighborhood, culture, values, spirituality that add to the complexity of its measurement [4].

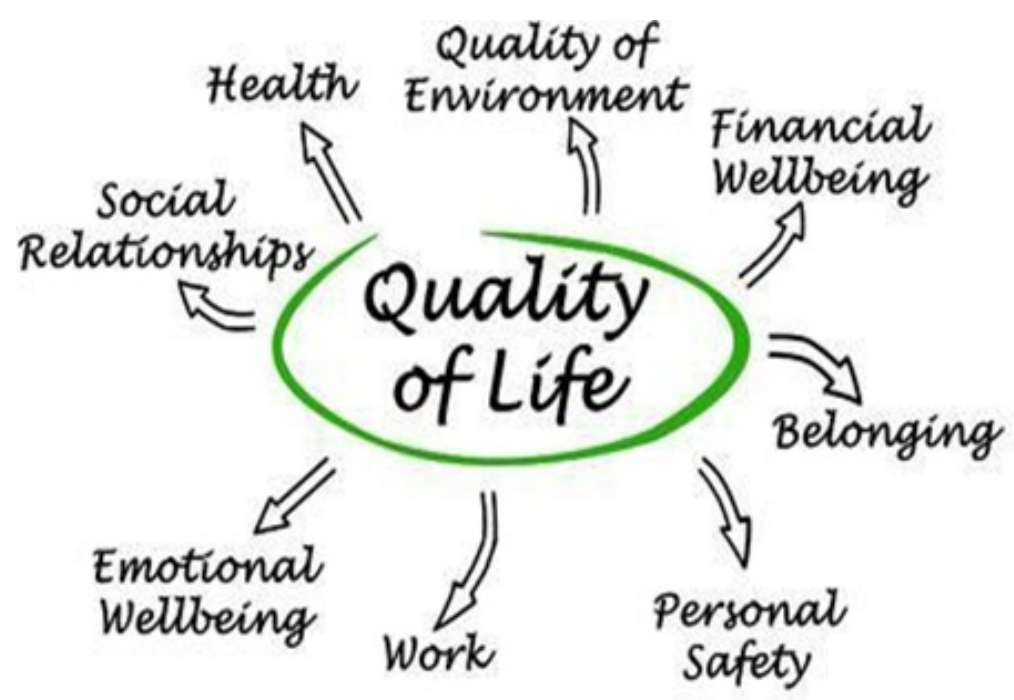

Figure 1: Source: https://www.123rf.com/photo_58468063_diagram-of-quality-of-life.html [6].

Academic interest in quality of life has mainly grown after World War II, when there was an increasing awareness and recognition about social inequalities. This setup has provided the major impetus for social indicators research and subsequently for research on subjective well-being and quality of life, also covering issues like feelings of loss of control, perceptions of social stigma, etc. [5]. Quality of life as evaluated by people is based on the horizons of possibilities that they see for themselves being a fundamental component of their identity. These horizons are further determined to various levels by factors such as social class, age, sex, ethnic group, sexuality, disability and personal say about one's life [7]. 


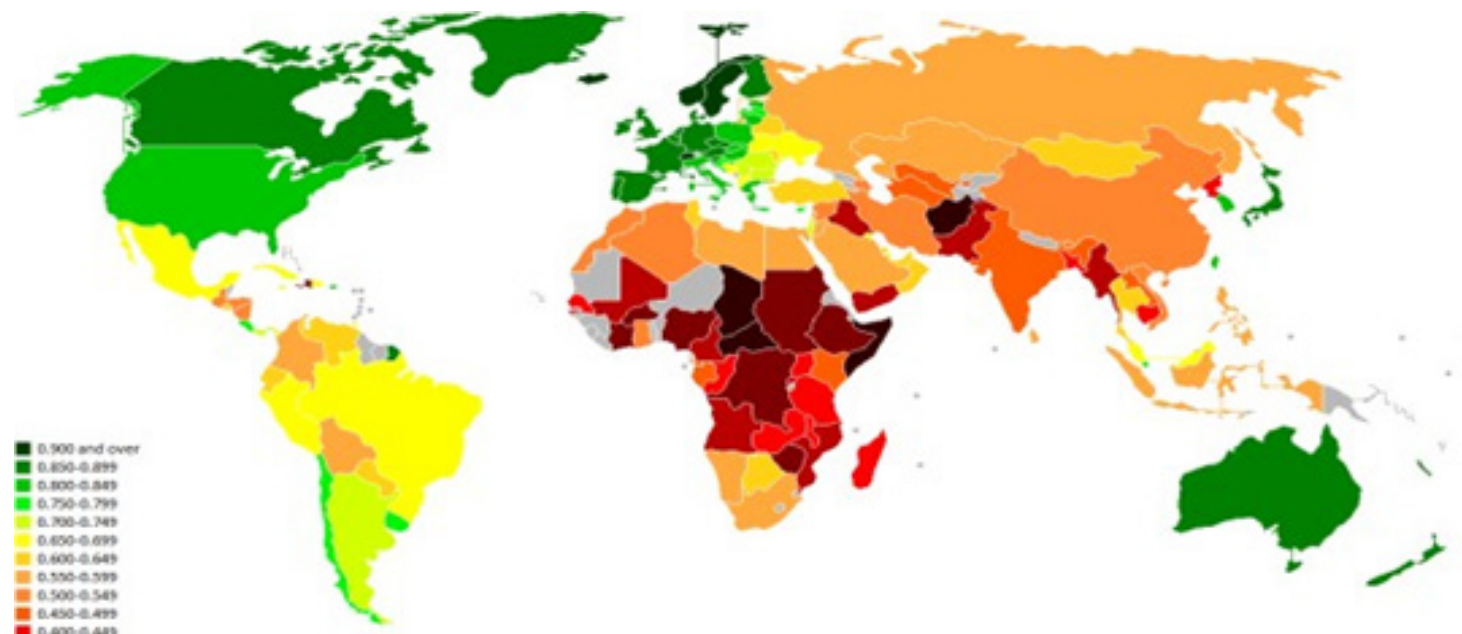

2011 Quality of Life Index

Figure 2: Source: https://nationranking.wordpress.com/category/quality-of-life-index/ [8]

With a score of $83 \%$, Denmark was followed by Germany (83.1\%), the Nordic quartet of Finland (82), Norway (81.7), Iceland (81.6) and Sweden (81.6) making up the top six nations with the highest scores for quality of life among their natives [9].

\section{Impact of Lifestyle Habits on Cognitive Function}

Deterioration in cognitive function, like physical decline, is a hallmark of ageing and predicts mortality $[10,11]$. Perceptions, memory, thinking, reasoning and consciousness are all examples of cognitive function [11]. Cadar D, et. al. [2] proved that consistently healthy dietary choices are associated with slower memory decline. The study also indicated that unremittingly sufficient level of physical activity during all midlife starting from a much earlier phase contributes toward slower visual search speed decline, independent of lifestyle behaviour, social class of origin, childhood cognition, educational attainment, adult social class, as well as symptoms of anxiety and depression. Smoking was not associated with cognitive outcome as per the study. Physically active lifestyle is known to impact executive control, processing speed and controlled processing, positively. Promoting a physically active lifestyle throughout adulthood may help to slow the deterioration of effortful executive control functions as people age [12]. Nicotine affects a wide range of cognitive domains, including sensorial, motor, attention, executive function, learning and memory due to the widespread availability of nicotinic acetylcholine receptors (nAChRs) throughout the brain in addition to distressing a wide range of neurotransmitter systems (norepinephrine, serotonin, and dopamine). Smoking reinforcement could be attributed to the first cognitive benefit, in which people learn that smoking improves cognitive performance briefly (improving some components of attention and memory). These acute nicotine effects improve cognitive performance above that of non-smokers and cognitive disturbance caused by nicotine withdrawal. Both neurobiological effects operate as reinforcers for nicotine use, accelerating the onset of nicotine addiction. However, heavy smoking is associated with cognitive impairment and its resulting decline mainly in the middle age [13]. Although stopping smoking at any time appeared to be beneficial, the risk of dementia was linked to the length of time since quitting. The necessity of quitting smoking in forties and fifties to reduce the risk of dementia has been emphasized [14].

\section{Impact of Lifestyle Diseases on Cognitive Function}

Nowadays, lifestyle diseases are becoming more common, affecting especially the young and urban-dwelling population $[15,16]$. The median age group at risk is indeed shifting from the fifth decade to perhaps the fourth decade of life or even younger inhabitants. Sedentary lifestyle or a lack of sufficient level of physical exercise is one of the main causes of preventable mortality, globally [16]. Physiologicallystressful lifestyle results into increased levels of risk factors, like hypertension or high blood pressure, dyslipidemia which refers to the abnormal amount of lipids mainly cholesterol or fats in the bloodstream, high blood glucose, respiratory 
diseases and abnormal or excessive body fat accumulation, heart-related diseases; acting altogether synergistically on various body metabolic pathways [15-18]. Many developing nations are typically burdened with lifestyle-related diseases (along with other communicable diseases) [16,19]. India has already been considered as 'the diabetes capital of the world' [16].
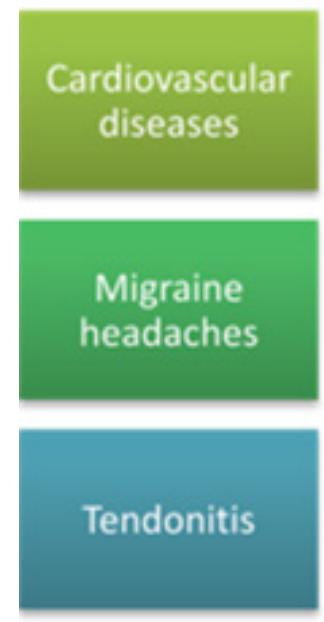
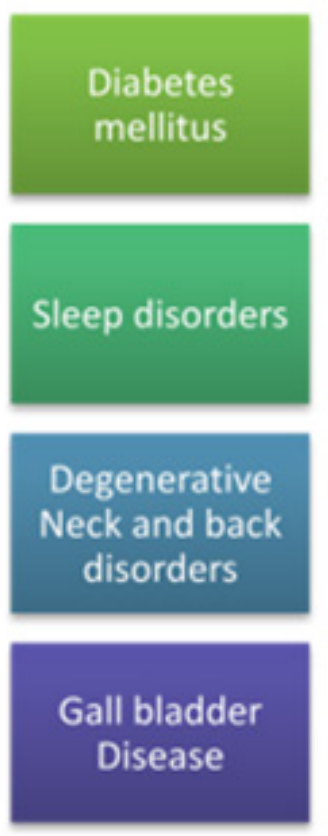
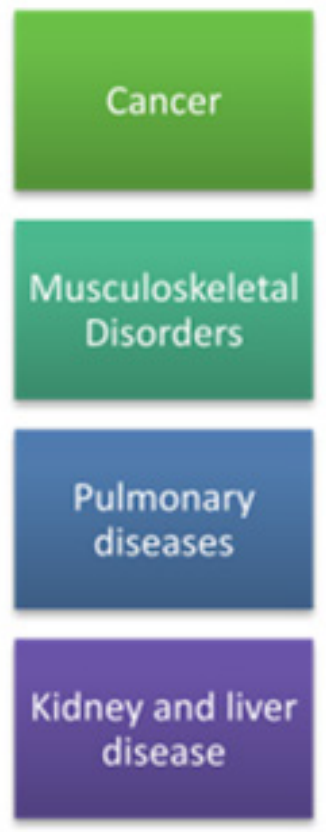
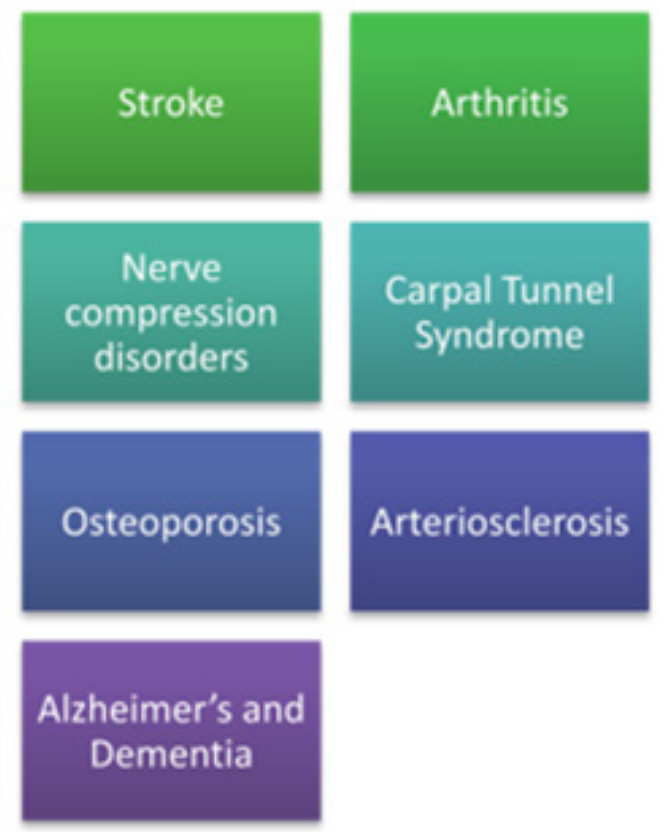

Figure 3: List of common lifestyle disorders. Source: http://ergo-plus.com/wp-content/uploads/Causes-of-Lifestyle-Diseases. pdf [20].

Cherubini A, et al. [21] elucidated that high blood pressure causes different types of cerebral vascular damage. This is associated with an increase in atherosclerosis in the larger vessels and in the oxidative stress at the level of the vascular wall degenerative central nervous system (CNS) disease, which manifests clinically as dementia (such as Alzheimer's disease) or vascular dementia (a long-term consequence of hypertension), is more likely to develop as the population ages. Hypertensive people are more likely to have brain atrophy. A high number of neurofibrillary tangles (NFT) have also been reported. These findings may help to explain why there is often a link between hypertension and the degenerative dementia of Alzheimer's disease [21].

One study aimed to examine the cognitive performance of older male prisoners and its effect on their perceived health and quality of life. It has been found that there were very significant differences between the two groups in terms of cognitive performance, perceived health and quality of life. There did not exist any significant association between cognitive impairment and perceived health or quality of life. Prisoners suffering from cognitive impairment do not necessarily seek help, perhaps not even considering that their health or quality of life to be affected [22]. Obesity is becoming more common in Europe, which is linked to impaired glucose tolerance (IGT), type 2 diabetes mellitus and low-grade inflammation, all of which are linked to cognitive impairment in the short and long term, culminating in metabolic syndrome. As a result, obesity and its comorbidities are risk factors for poor cognitive function and cognitive decline [23]. A negative relationship has been shown between anthropometric measures of obesity (such as BMI, waist circumference) and a variety of cognitive areas. Obesity, for instance, is linked to poor performance on episodic memory tasks. Verbal learning is hindered in people with a greater BMI versus those with a lower BMI, as measured by delayed recall and identification of words. For visual modality episodic memory tests, similar losses have been found. Sub-standardized eating disorders such as anorexia nervosa and bulimia result in decisionmaking problems. Obese people's decision-making abilities have been demonstrated to be at risk in a number of studies. Adiposity is linked to a variety of health problems, including vascular comorbidities, which raise the risk of dementia.

Insulin resistance has been linked to chronic, low-grade inflammation associated with obesity. It is central to the 
metabolic syndrome, which has been associated with an increased risk of cognitive decline and dementia. Insulin resistance, predictive of subsequent development of type 2 diabetes mellitus, not only raises the likelihood of subsequent cognitive impairment, but it may also hasten the ageing of the neurons [23]. Lower age, greater fitness (push-up strength, VO(2)max, and activity duration during treadmill testing), and lower fasting glucose levels were associated with improved Neurocognitive Index (NCI) scores. Younger age, lower systolic blood pressure, lower body fat, lower carotid IMT scores, greater fitness, and more alcohol intake were also associated with better cognitive flexibility scores. Modifiable extrinsic factors impacting cognitive performance favorably include greater aerobic fitness and strength, lower blood glucose levels, greater alcohol intake, lower body fat and avoidance of tobacco. However, further studies are still needed to investigate and ascertain whether modifying these lifestyle
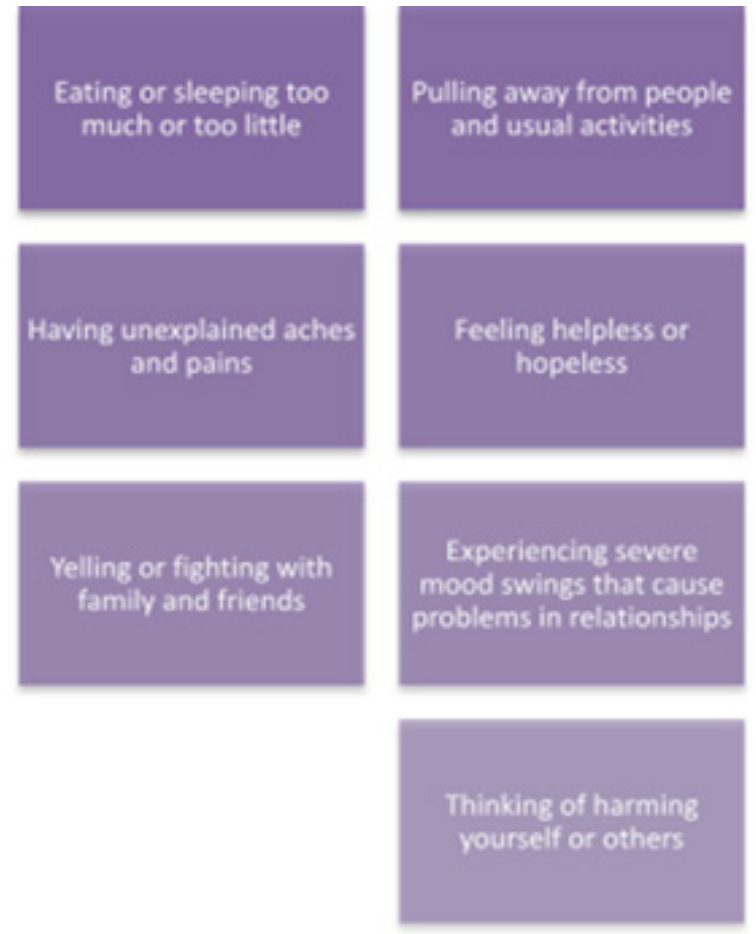
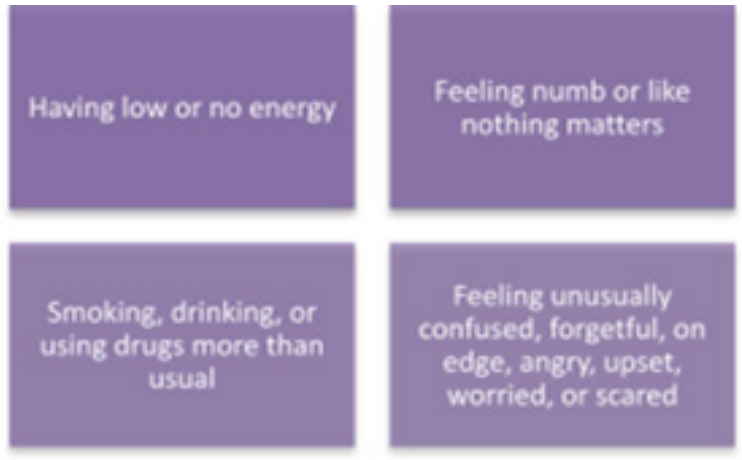

Having persistent thoughts and memories vou can't get out of your head
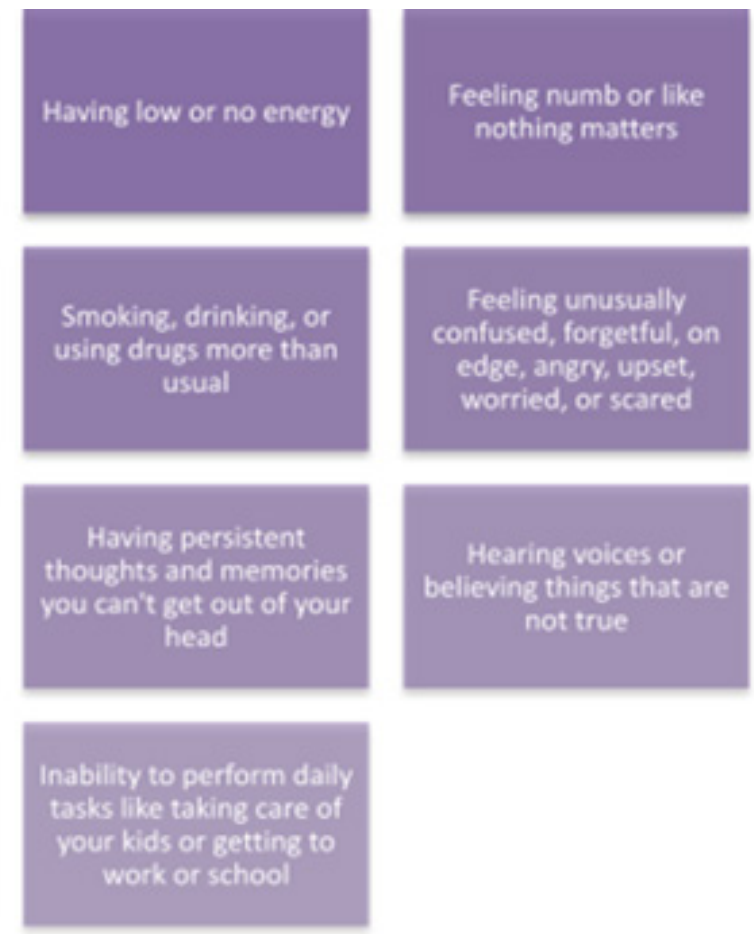

factors improves cognitive function and slows cognitive decline. Greater aerobic fitness and strength, lower blood sugar levels, increased alcohol intake, lower body fat, and cigarette avoidance are all modifiable biomarkers that have a positive impact on cognitive performance. More research is needed to see if changing these lifestyle factors might improve cognitive performance and slow cognitive decline [24].

Certainly, the urbanization process has caused poor lifestyle choices (such as smoking, overuse of alcohol, poor nutrientrich diet, insufficient physical activity and chronic stress) leading to the development and progression of various chronic diseases. Even though doctors and social media are there to encourage practicing healthy habits to help prevent most of these chronic medical disorders, people generally ignore or are reluctant to either start or maintain the appropriate and healthy behavior [16].

Figure 4: Early signs of poor mental health. Source: https://www.mentalhealth.gov/basics/what-is-mental-health [25]. 
Poor Mental Health, Cognitive Impairment and Quality of Life

Mental health is important at every stage of life (from childhood and adolescence through adulthood), that considers emotional, psychological and social well-being of an individual [25]. The promotion and protection of good mental health is becoming a major societal concern; since good mental health is associated with greater economic success, better social relationships and reduced risk of poor health and illnesses. [11,26]. It affects and determines how one thinks, feels, acts, handles stress, relates to others and makes choices as well as behavior. Several factors play a significant role in the development of mental health problems, which include biological factors, such as genes or brain chemistry; life experiences, such as trauma or abuse; and family history of mental health problems [25].

Independence in later life is as much determined by cognitive ability as by physical self-dependency [11,27]. Referring to the older adults, a broad spectrum of cognitive capability exists with dementia at one end and normal cognitive functioning at the other. Simple activities of daily living such as dressing and bathing, and more complex tasks such as managing money, paying bills, taking medications, ability to work, playing a role and decision-making can only be performed with an adequate level of cognitive function. Modifiable risk factors and early interventions to prevent cognitive decline and dementia are key priorities for the policy-makers and societies right now. Despite its importance there is a lack of adequate data sources to derive population-based estimates of the prevalence and consequences of cognitive impairment over the lives of the elderly. It is increasingly important to investigate and understand the mechanisms to promote optimal cognition functioning with advancing age for a much larger proportion of the population would then be suffering from dementia and other related aspects sooner or later [11].

Measurement of quality of life is a very complex process. Researchers are still confused about the factors which determine the quality of life. Many researches have been done so far for evaluating the appropriate method which increased the complexity more. A theoretical model for measuring quality of life has been developed which clearly explains the external and internal factors. According to scientific literatures, the primary elements impacting quality of life that have been recognized in many sectors of science are external elements include freedom, political stability, economic environment, educational accessibility, and social security; internal elements include health, personal security, educational achievement, family, income, and housing [28]. Overall, the research suggests that lifestyle aids in the promotion of healthy cognitive functioning and effective ageing. The findings were comparable across different groups with varying characteristics in areas including sex, ethnicity, environment and diagnosis. A study showed relevant patientimprovement initiatives, psycho-education could involve stress management and relaxation skills to assist individuals cope with unpleasant thoughts, feelings, and emotions, while negative disease perceptions were linked to poor health related quality of life [29]. The most recent studies continue to indicate a positive association between spirituality and quality of life. The most studied patients are oncological (26 percent), with chronic diseases (17 percent) and mental illnesses (8 percent). In the general population, the most studied groups are caregivers, elderly, and students. There is consistent literature supporting the existence of a positive relationship between spirituality and quality-of-life [30].

\section{Impact of Environment on Cognitive Impairment and Quality of Life}

A study has shown the effects of the natural environment on human cognitive function and mental health, synthesizing work from environmental psychology, urban planning, the medical literature and landscape aesthetics, closely related to memory, attention, concentration, impulse inhibition, and mood. A strong favorable link between urban green space exposure (including gardens) and physical and emotional wellbeing has been shown in several observational studies [31]. Early and midlife circumstances may influence late-life cognitive outcomes, according to a life course epidemiology view, in part through altering lifestyle patterns and social and economic factors. Demographic markers that are linked to latelife cognition, such as education and ethnicity, are complicated variables that are intertwined with socioeconomic status (SES) and a slew of other disparities in childhood and adult life events. 


\section{Ways to maintain positive mental health}

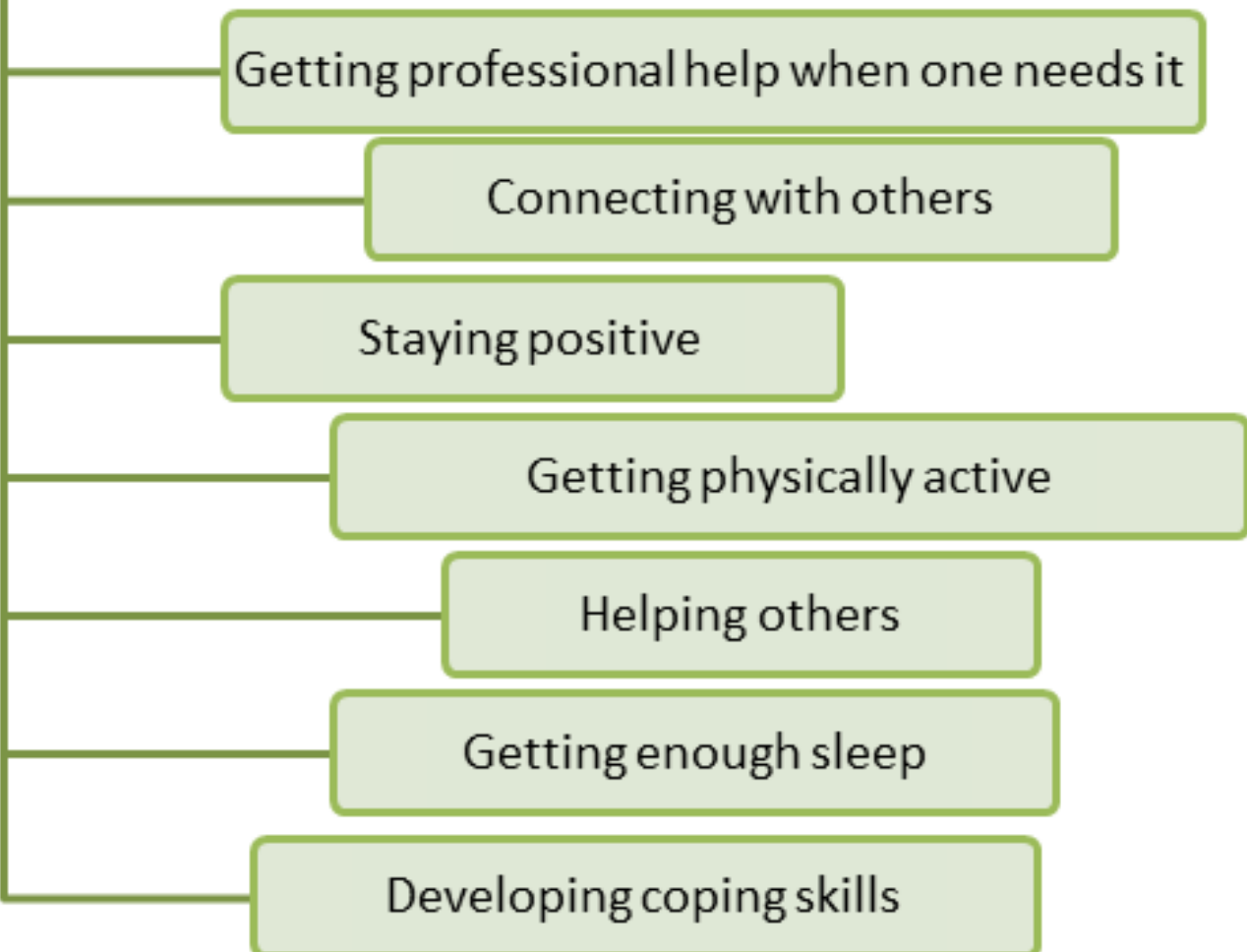

Figure 5: Source: https://www.mentalhealth.gov/basics/what-is-mental-health [25].

The effects of age and APOE on cognitive decline are expected to be mediated through well-known connections with ageing disorders such as Alzheimer's disease and cerebrovascular disease. Single word reading, on the other hand, has long been thought to be a good sign of educational quality, and hence could be a good sign of life experience that influences cognitive ageing. There was a link between higher levels of current recreational activities and less cognitive deterioration. Recreational pursuits in later life have been linked to cognitive deterioration. There are several possible causes for these correlations. Early life experiences that create resilience to diseases that induce cognitive decline may be a factor of late-life activity. In the final multivariate model, heavy physical activity at age 40 was linked to better baseline cognition, which is consistent with previous findings linking leisure physical activity in midlife to a lower risk of dementia, Alzheimer's disease, and vascular dementia, as well as improved processing speed, memory, and executive function. The impacts of language, race/ethnicity, and education on cognition have been thoroughly documented in cross-sectional studies [32].

Patients with anxiety disorders and low quality of life outnumbered those in the control group who used negative coping strategies to deal with stressful situations regardless of gender, age, occupation, education, marriage, religious belief, or other factors [33]. A high level of adherence to the Mediterranean diet has been linked to a slower rate of cognitive decline, a lower risk of acquiring MCI, a lower risk of MCI conversion to Alzheimer's disease, and a lower risk of Alzheimer's disease [34]. Physical activity plays a major role. Although the mechanisms behind the observed acute aerobic exercise effects on cognitive performance may be age dependent, light and moderate aerobic exercise improves cognitive function through the adult lifespan [35].

\section{Genetic Impact on Cognition and Quality of Life}

Higher serum phosphate levels were linked to poor executive performance in older males, but not to impaired global 
cognitive performance or decrease in executive or global cognition [36]. High initial interleukin (IL-6) concentrations in the plasma of older people were found to be predictive of eventual disability, including cognitive impairment, in a longitudinal research [37]. Previous research suggested that the negative effect of smoking on the development of dementia was limited to people who didn't have the APOE 4 allele. However, when looked into cognitive decline, no indication of a difference in the rate of cognitive decline based on the APOE 4 genotype was found [38].

\section{CONCLUSION}

Human variations do not hold good with universal solutions applied to different concerns globally. Understanding the dynamics of crossover between the biological and cultural aspects of living and nature of matter, having humans at its core/center, determine a wide range of solution. With this, addressing the subject-matter using a community-based approach is much desirable. Upon which a community feedback is an added point to the whole process of social research. Highly motivated caregivers working towards attending, supporting and most importantly understanding the immediate health-destitution of patients is the chief demand to be addressed right away.

Holistic nature and subject matter of Anthropology encompasses evaluating multiple possibilities and reasons for a condition. Then, talking about neuroanthropology seems incomplete without making mention of aspects like quality of life, mental health and cognition in further relation to lifestyle related disorders that are so complexly intertwined. Domains of all these subsets seem to possess different paradigms, however, their influence meet upon the same base-human, thereby overlapping in terms of repercussions arising from undesirable undertakings. Lifestyle-relating behavior and experiences have outcomes on health- both physical and mental, regulating growth, development and maturation as a result. This in turn has a drastic influence on the perception about the quality of living. Considering this complex web of association and action among these parameters is the real essence of the umbrella concept of neuroanthropology which looks at various possibilities of manifestation of a condition at a particular point in time. Researchers should focus on measuring quality of life in relation to the aforesaid factors for reliability and accuracy of research.

\section{ACKNOWLEDGEMENTS}

The author is highly grateful to the 4th International Conference on Neurology and Brain Disorders for providing the platform to discuss the respective sub-theme.

\section{Authors' Contributions}

Conception of idea - MD; Preparation of manuscript - UG, SJ; Proofreading - MD, UG.

\section{CONFLICTS OF INTEREST}

Authors declare that they have no conflict of interest.

\section{REFERENCES}

1. Lende DH, Downey G. (2012). Neuroanthropology and its applications: an introduction. Annals of Anthropological Practice. 36(1):1-25

2. Cadar D, Pikhart H, Mishra G, Stephen A, Kuh D, Richards M. (2012). The role of lifestyle behaviors on 20-year cognitive decline. Journal of aging research. 304014.

3. https://www.who.int/toolkits/whoqol

4. https://www.cdc.gov/hrqol/concept.htm

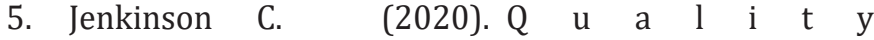
of life. Encyclopedia Britannica. https://www. britannica.com/topic/quality-of-life

6. https://www.123rf.com/photo_58468063_diagram-ofquality-of-life.html

7. Carr AJ, Gibson B, Robinson PG. (2001). Is quality of life determined by expectations or experience?. Bmj. 322(7296):1240-3.

8. https://nationranking.wordpress.com/category/qualityof-life-index/

9. https://studyindenmark.dk/news/denmark-has-thesecond-best-quality-of-life-in-the-world

10. Dewey ME, Saz P. (2001). Dementia, cognitive impairment and mortality in persons aged 65 and over living in the community: a systematic review of the literature. Int J Geriatr Psychiatry. 16(8):751-61.

11. https://tilda.tcd.ie/publications/reports/pdf/w1-keyfindings-report/Chapter6.pdf

12. Ballesteros S, Mayas J, Reales JM. (2013). Does a physically active lifestyle attenuate decline in all cognitive functions in old age? Curr Aging Sci. 6(2):189-198. doi:10.2174/18 


\section{1.}

13. Waisman Campos M, Serebrisky D, Mauricio CastaldelliMaia J. (2016). Smoking and cognition. Current drug abuse reviews. 9(2):76-9.

14. Deal JA, Power MC, Palta P, Alonso A, Schneider AL, et al. (2020). Relationship of cigarette smoking and time of quitting with incident dementia and cognitive decline. Journal of the American Geriatrics Society. 68(2):337-45.

15. WHO, 2011. Global status report on non-communicable diseases 2010. World Health Organization http://www. who.int/nmh/publications/ ncd_report_full_en.pdf

16. Senapati S, Bharti N, Bhattacharya A. (2015). Modern lifestyle diseases: chronic diseases, awareness and prevention. Int J Curr Res Acad Rev. 3(3):215-3.

17. Rosenstock L, Cullen M, Fingerhut M. (2006). Occupational health. Disease Control Priorities in Developing Countries. 2nd edition.

18. Willett WC, Koplan JP, Nugent R, Dusenbury C, Puska P, Gaziano TA. (2006). Prevention of chronic disease by means of diet and lifestyle changes. Disease Control Priorities in Developing Countries. 2nd edition.

19. Pappachan MJ. (2011). Increasing prevalence of lifestyle diseases: high time for action. The Indian journal of medical research. 134(2):143.

20. http://ergo-plus.com/wp-content/uploads/Causes-ofLifestyle-Diseases.pdf

21. Cherubini A, Lowenthal DT, Paran E, Mecocci P, Williams LS, et al. (2010). Hypertension and cognitive function in the elderly. Disease-a-month. 56(3):106-47.

22. Combalbert N, Pennequin V, Ferrand C, Armand M, Anselme M, et al. (2018). Cognitive impairment, selfperceived health and quality of life of older prisoners. Criminal Behaviour and Mental Health. 28(1):36-49.

23. Dye L, Boyle NB, Champ C, Lawton C. (2017). The relationship between obesity and cognitive health and decline. Proceedings of the nutrition society. 76(4):44354.

24. Masley SC, Roetzheim R, Clayton G, Presby A, Sundberg K, et al. (2017). Lifestyle markers predict cognitive function. Journal of the American College of Nutrition. 36(8):61723.
25. https://www.mentalhealth.gov/basics/what-is-mentalhealth

26. Cornwell K, Forbes C, Inder B, Meadows G. (2009). Mental illness and its effects on labour market outcomes. J Ment Health Policy Econ. 12(3):107-18.

27. Greiner PA, Snowdon DA, Schmitt FA. (1996). The loss of independence in activities of daily living: the role of low normal cognitive function in elderly nuns. American Journal of Public Health. 86(1):62-6.

28. Pukeliene V, Starkauskiene V. (2011). Quality of life: Factors determining its measurement complexity. Engineering Economics. 22(2):147-56.

29. Fanakidou I, Zyga S, Alikari V, Tsironi M, Stathoulis J, et al. (2018). Mental health, loneliness, and illness perception outcomes in quality of life among young breast cancer patients after mastectomy: the role of breast reconstruction. Quality of Life Research. 27(2):539-43.

30. Panzini RG, Mosqueiro BP, Zimpel RR, Bandeira DR, Rocha NS, et al. (2017). Quality-of-life and spirituality. International Review of Psychiatry. 29(3):263-82.

31. Bratman GN, Hamilton JP, Daily GC. (2012). The impacts of nature experience on human cognitive function and mental health. Annals of the New York academy of sciences. 1249(1):118-36.

32. Brewster PW, Melrose RJ, Marquine MJ, Johnson JK, Napoles A, et al. (2014). Life experience and demographic influences on cognitive function in older adults. Neuropsychology. 28(6):846.

33. Dong M, Zhang JX, Lu CH, Tang JS, Liu LF, et al. (2013). A case-control study on the quality of life and the way of response among patients with anxiety disorder in Shandong province. Zhonghua liu xing bing xue za zhi Zhonghua liuxingbingxue zazhi. 34(10):953-7.

34. Féart C, Samieri C, Barberger-Gateau P. (2010). Mediterranean diet and cognitive function in older adults. Current opinion in clinical nutrition and metabolic care. 13(1):14

35. Kamijo K, Hayashi Y, Sakai T, Yahiro T, Tanaka K, et al. (2009). Acute effects of aerobic exercise on cognitive function in older adults. Journals of Gerontology: Series B. 64(3):356-63. 
36. Slinin Y, Vo T, Taylor BC, Murray AM, Schousboe J, et al. (2008). Osteoporotic Fractures in Men (MrOS) Study Group. Serum phosphate and cognitive function in older men. International journal of geriatric psychiatry. 33(1):159-66.

37. Bodles AM, Barger SW. (2004). Cytokines and the aging brain-what we don't know might help us. Trends in neurosciences. 27(10):621-6.
38. Wingbermühle R, Wen KX, Wolters FJ, Ikram MA, Bos D. (2017). Smoking, APOE genotype, and cognitive decline: the Rotterdam study. Journal of Alzheimer's Disease. 57(4):1191-5. 